\title{
Energy Harvesting and Information Transmission Protocol in Sensors Networks
}

\author{
Xue-Fen Zhang ${ }^{1}$ and Chang-Chuan Yin ${ }^{2}$ \\ ${ }^{1}$ College of Information Technology, Beijing Union University, Beijing 100101, China \\ ${ }^{2}$ Key Laboratory of Universal Wireless Communications, Ministry of Education, Beijing University of Posts and Telecommunications, \\ Beijing 100876, China
}

Correspondence should be addressed to Xue-Fen Zhang; zhangxuefen@buu.edu.cn

Received 17 June 2015; Revised 2 September 2015; Accepted 7 September 2015

Academic Editor: Gwanggil Jeon

Copyright (C) 2016 X.-F. Zhang and C.-C. Yin. This is an open access article distributed under the Creative Commons Attribution License, which permits unrestricted use, distribution, and reproduction in any medium, provided the original work is properly cited.

\begin{abstract}
We focus on the design of transmission protocol for energy harvesting wireless sensors. The sensors can harvest the energy from the environment, but they cannot charge and discharge at the same time. We propose a protocol for energy harvesting and wireless transmission, which contains two steps. In the first step, the sensor harvests the energy from environment, and the energy harvesting rate is controlled by the harvested energy power of the energy saving device (ESD). In the second step, some data should be transmitted to the receiver in a certain time. Considering one slot time, the first part of the time is devoted exclusively to energy harvesting, and the remaining time of the slot is for transmitting the information data. Assume that $Q$ bits are transmitted to the receiver within one time slot; we establish the relationship between the harvesting energy time and the transmit data time. In addition, we analyze the system outage probability performance over the Rayleigh fading channel.
\end{abstract}

\section{Introduction}

The energy harvesting in wireless sensor network has become increasingly attractive with sustainable and long lasting power supplies. With development of IOT, more and more sensors are embedded in medical device or building structures to sense the environment [1-3]. Sensor nodes are powered by batteries that often cannot be replaced because of the inaccessibility of the devices (e.g., inside the human body). Once the battery of a sensor node is exhausted, the node dies. In this case, a solution may be to harvest the energy from the environment. The energy harvesting methods include absorbing energy from the solar wind, vibration, RF radio waves, temperature differences, and airflow, thermoelectric effects, and other physical phenomena [4].

Among all these methods, radio signal radiated by ambient transmitters became a new source for wireless energy harvesting, and harvesting energy from ambient RF signals can power a wireless sensors network [5]. Besides, recent advance in designing highly efficient rectifying antennas will enable more efficient wireless energy harvesting from RF signals in the near future $[6,7]$. More recently, the study of wireless powered communication networks (WPCNs) has received a lot of interest. For a WPCN, the energy harvested from ambient RF signals is used to power wireless terminals in the network [8]. In addition, the advantage of RF solution lies in the fact that RF signals can carry energy and information at the same time, and such an approach can also reduce the cost of communication networks, since peripheral equipment to take advantage of external energy sources can be avoided. Besides, ambient RF from TV and cellular communications is widely available in urban areas (day and night, indoors and outdoors) [9].

There is some progress in the recent research of energy harvesting. For example, the information theoretic capacity with an energy harvesting transmitter was discussed in [10]. The paper introduced two different capacity-achieving schemes and concluded that the capacity of an energy harvesting AWGN channel is the same as that of a traditional AWGN channel as long as the average available energy is the same. In [11], the problem of transmission time minimization in an energy harvesting setting for a point-to-point 
communication system was studied. In $[12,13]$, the authors investigated the minimization of the transmission time for a given amount of data through power control based on known energy arrivals over all the time. In [14], the authors considered the power allocation for an access-controlled transmitter with energy harvesting capability based on causal observations of the channel fading state. In addition, the problem of transmission time minimization was also solved by mapping it to the problem of throughput maximization.

In this work, we consider the design of transmission protocol for energy harvesting wireless sensor system, in which the users transmit their independent information using their individually harvested energy in the uplink. Our work is unlike traditional research on Simultaneous Wireless Information and Power Transfer (SWIPT), which assumes the simultaneous energy and information transmissions to sensors (or users) in the downlink. A major problem of this assumption is that practical circuits cannot charge and discharge at the same time. We assume that the transmitter needs to deliver $Q$ bits within $T$ seconds, where $T$ is the duration of a transmission frame. The transmitter can only harvest energy or deliver information at any given time, but not both. As a result, the transmitter needs to decide when to switch between the transmission data (TD) mode and the energy harvesting $(\mathrm{EH})$ mode. We derive the optimal mode switching rule at the transmitter to achieve various tradeoffs between the minimum transmission outage probability in TD mode and the average harvested energy in $\mathrm{EH}$ mode.

The rest of this paper is organized as follows. Section 2 provides the system model. We analyze the outage probability in Section 3 and establish the optimization problem in Section 4. Section 5 presents numerical examples. Finally, Section 6 concludes the paper.

\section{System Model}

In this paper, we assume the system includes one pair of single-antenna transmitter $(\mathrm{Tx})$ and receiver $(\mathrm{Rx})$. Suppose $Q$ bits are transmitted to the receiver within $T$ seconds, where $T$ is the duration of a transmitter frame. In every frame, there are two phases of transmission. The first phase is called harvesting time, in which ESD collects the energy from the environment. Since the ESD cannot charge and discharge simultaneously, the second step is for the data transmission in the remaining frame time. Next, we give the key parameters in the communication protocol for energy harvesting and information processing at Tx. Define the save-ratio $\rho$ as the fraction of the one transmission slot time, and, during the time interval $[0, \rho T]$, Tx harvest energy by ESD. The remaining slot time, from time $\rho T$ to $(1-\rho) T$ seconds, is used for the information transmission. In this paper, the relation between $\rho$ and outage probability is established.

Let $h_{s, d}$ be the source-destination channel coefficients. The channel from the source to the destination is modeled as frequency nonselective fading and additive noise $[1,5,7]$. Therefore, the channel coefficients are assumed to be constant during a complete slot time and may vary from a slot time to another.
The receiver signal at the destination node in one slot time, $y_{r}(t)$, can be written as

$$
y_{r}(t)=\sqrt{\frac{P_{s}}{d_{s, d}^{\eta}}} h_{s, d} x(t)+n_{s, d},
$$

where $h_{s, d}$ is the source-to-receiver channel gain. We model $h_{s, d}$ as independent zero-mean, circularly symmetric complex Gaussian random variables with variances $\sigma_{s, d}^{2}$, the envelope of which follows Rayleigh distribution (for Rayleigh fading the channel amplitude squares $\left|h_{s, d}\right|^{2}$ are exponentially distributed). The noise is modeled as independent zeromean, circularly symmetric complex Gaussian random variables with zero means and variances. $d_{s, d}$ is the source-toreceiver distance, $P_{s}$ is the average power transmitted from the source in the time slot, $\eta$ is the path loss exponent, and $x(t)$ is the source message with unit power; that is, $E\left[|x(t)|^{2}\right]=1$, where $E[\cdot]$ is the expectation operator and $|\cdot|$ is the absolute value operator. $n_{s, d}$ denotes Gaussian noise with unit variance.

According to the protocol, the total buffered energy at $t=\rho T$, which is the time of start to transmit data within a transmission slot, is given by $E_{T}=P_{T} \rho T$, where $P_{T}$ is the harvested energy power of the ESD.

Denote $P_{s}=E_{T} /(1-\rho) T=P_{T}(\rho /(1-\rho))$ as the average total power, which is constant over the entire transmission period.

\section{Outage Probability}

3.1. SNR Analysis. In this section, we analyze the instantaneous signal-to-noise ratio (SNR) and derive the probability density function (PDF) of the SNR.

The instantaneous SNR in the destination is given by

$$
\gamma_{0}=\frac{P_{s}\left|h_{s, d}\right|^{2}}{d_{s, d}^{\eta} N_{0}},
$$

where $N_{0}$ is the variance of AWGN in the corresponding channels. We can see that the random variable $\gamma_{0}=P_{s}\left|h_{s, d}\right|^{2} /$ $d_{s, d}^{\eta} N_{0}$ is an exponential random variable with parameter $\beta_{0}=N_{0} d_{s, d}^{\eta} / P_{s} \sigma_{s, d}^{2}$. Hence, the PDF of $\gamma_{0}$ is

$$
f_{\gamma_{0}}(x)= \begin{cases}\beta_{0} e^{-\beta_{0} x}, & x>0 \\ 0, & \text { otherwise }\end{cases}
$$

3.2. Outage Probability Performance Analysis. For a given $h$, the cluster-to-cluster amplify-and-forward relay network's mutual information is

$$
I=\log _{2}\left(1+\gamma_{0}\right) .
$$

The outage probability at $\mathrm{Rx}$ can be expressed as

$$
\begin{aligned}
P_{\text {out }} & =\operatorname{Pr}\{I<R\}=\operatorname{Pr}\left\{\log _{2}\left(1+\gamma_{0}\right)<R\right\} \\
& =\operatorname{Pr}\left\{\gamma_{0}<2^{R}-1\right\},
\end{aligned}
$$

where $R$ is the required target rate. 
Because

$$
f_{\gamma_{0}}(x)= \begin{cases}\beta_{0} e^{-\beta_{0} x}, & x>0 \\ 0, & \text { otherwise }\end{cases}
$$

hence

$$
\begin{aligned}
P_{\text {out }} & =\operatorname{Pr}\left\{\gamma_{0}<2^{R}-1\right\}=\int_{0}^{2^{R}-1} f_{\gamma_{0}}(x) d_{x} \\
& =\int_{0}^{2^{R}-1} \beta_{0} e^{-\beta_{0} x} d_{x}=1-e^{-\beta\left(2^{R}-1\right)} .
\end{aligned}
$$

\section{Optimization Problem}

The outage probability is an important criterion of the system performance. In this section, we determine an optimal saveratio to improve the performance of outage probability. Suppose $Q$ bits of data are generated and transmitted within a time slot of duration $T$ seconds, and, for a given save-ratio $\rho$, the data delivery only takes place in the last $(1-\rho) T$ seconds of each time slot, which results in an effective rate of $R \geq R_{\text {eff }}=Q /(1-\rho) T$ bits/sec. In this section, we study the relation between $\rho$ and outage probability.

Consider the following optimization problem:

$$
\min P_{\text {out }}
$$

where $\rho \in(0,1)$.

According to (7), the optimization problem can be written as

$$
\min _{\rho \in(0,1)}\left(1-e^{-\beta\left(2^{R_{\mathrm{eff}}}-1\right)}\right) \text {. }
$$

According to $R \geq R_{\text {eff }}=Q /(1-\rho) T, \beta_{0}=N_{0} d_{s, d}^{\eta} / P_{s} \sigma_{s, d}^{2}$, and $P_{s}=E_{T} /(1-\rho) T=P_{T}(\rho /(1-\rho))$, we can rewrite the problem as

$$
\begin{aligned}
& \min _{\rho \in(0,1)}(1 \\
& \left.\quad-\exp \left(-\frac{N_{0} d_{s, d}^{\eta}}{P_{T}(\rho /(1-\rho)) \sigma_{s, d}^{2}}\left(2^{\mathrm{Q} /(1-\rho) T}-1\right)\right)\right) .
\end{aligned}
$$

Hence, the optimum $\rho$ is given by

$$
\min _{\rho \in(0,1)} \frac{(1-\rho) N_{0} d_{s, d}^{\eta}}{P_{T} \rho \sigma_{s, d}^{2}}\left(2^{\mathrm{Q} /(1-\rho) T}-1\right) .
$$

For (11), we can find the numerical solution based on golden section search and parabolic interpolation techniques [15].

\section{Numerical Examples}

In this section, we evaluate the performance of the proposed transmission schemes. First, we will calculate the mutual information $I$ and the outage probability versus the save-ratio

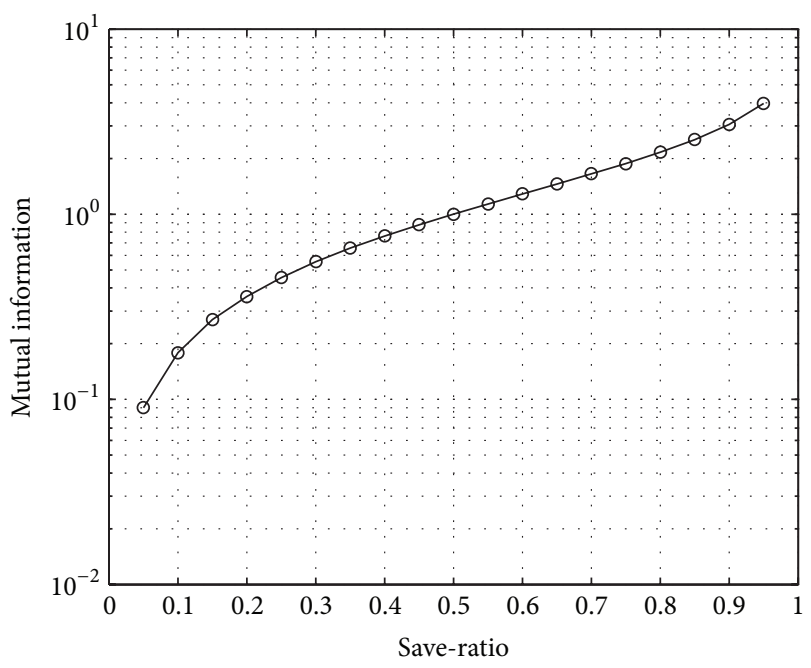

FIgURE 1: The system mutual information.

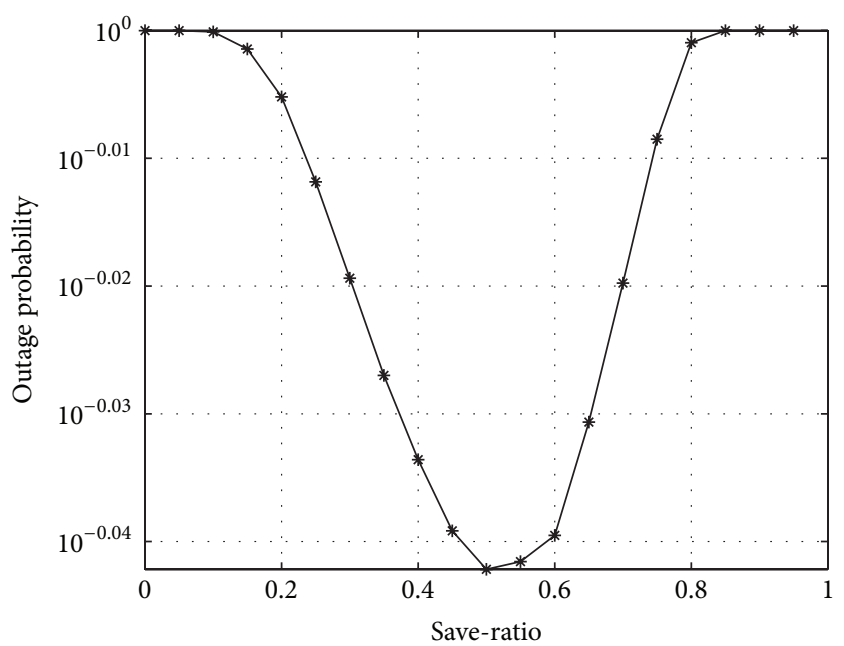

FIGURE 2: The relationship of outage probability and the save-ratio $\rho$.

$\rho$, respectively. Then, we will compute the minimum outage probability. Thus, we will derive the optimal save-ratio $\rho$.

In our simulation, we consider the BPSK modulation communication system. The channel is Rayleigh fading, and the path loss exponent $\eta$ is set as 2 . Without loss of generality, we assume that the source-to-receiver distance $d_{s, d}$ is normalized to 1 , and the channel between two nodes $h_{s, d}$ is i.i.d. Rayleigh fading random variables with unit variance. Besides, we also assume the target transmission information $Q=1$, and the time slot $T=1$. Hence, the target transmission rate $R \geq R_{\text {eff }}=Q /(1-\rho) T$ bits $/$ s.

Figure 1 shows the system mutual information versus the save-ratio. Figure 2 illustrates how the save-ratio $\rho$ affects the outage probability for $P_{T}=1$. As can be seen from Figure 2, the system mutual information $I$ is increased with the save-ratio $\rho$ changing from 0 to 1 , due to the improvement of instantaneous SNR in the destination. The save-ratio $\rho$ affects outage performance very significantly, which can be 


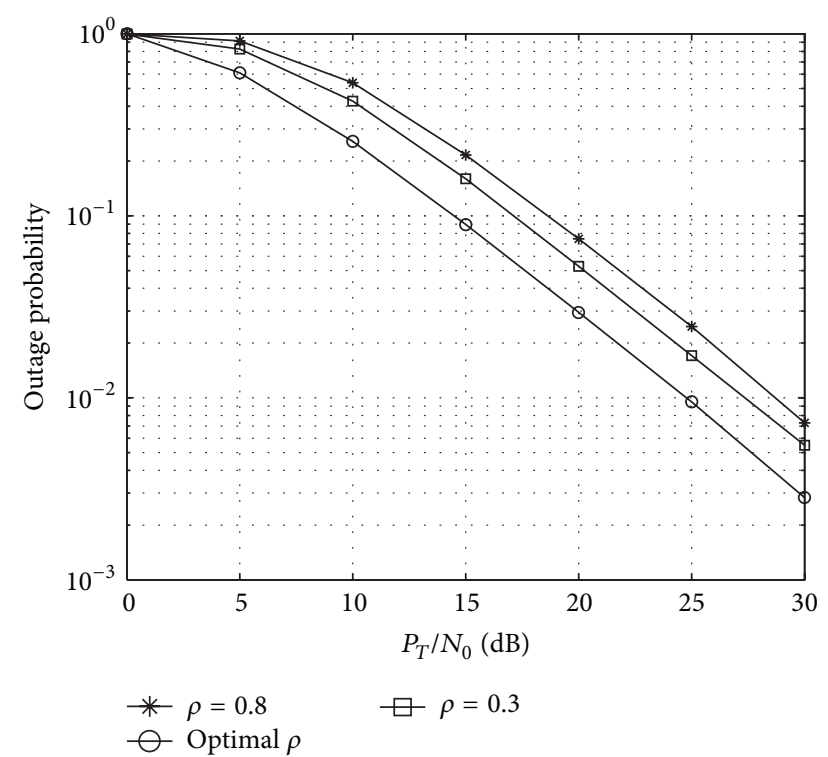

FIGURE 3: Outage probability comparison for optimal system and nonoptimal system.

seen from Figure 2. When the save-ratio is close to 0 , almost no energy is needed for the transmitter to send the data, so the SNR is low, and the outage probability is almost 1. If the save-ratio is close to 1 , though there is energy for the transmitter, the target transmission rate tends to $+\infty$. Hence, the outage probability is very high. As can be observed, there is an optimal decision on the save-ratio $\rho$ of the transmitters to achieve the optimal outage performance.

In Figure 3, we plot the outage probability for the optimal system. From this figure, we see that the optimal save-ratio $\rho$ scheme reduces the outage probability compared to the other cases.

\section{Conclusions}

In this paper, we studied a wireless system under energy harvesting conditions. We established the relationship between the system outage performance and the transmission protocol. Besides, we proposed an optimal method to optimize the system outage performance via finding the optimal saveratio. We also characterized how the outage probability varies with the save-ratio and compared the outage performance between nonoptimal save-ratio and optimal save-ratio under the assumption of Rayleigh fading channel. Simulation results demonstrated that remarkable gain is achieved for the proposed optimal scheme. If we choose the optimal save-ratio for the transmission, we can achieve the outage performance minimization. And the optimal scheme can provide improved efficiency and reliability of the energy harvesting wireless sensor networks, which means such energy harvesting approaches are promising for further performance improvement.

\section{Conflict of Interests}

The authors declare that there is no conflict of interests regarding the publication of this paper.

\section{Acknowledgments}

This work is partially supported by the National Natural Science Foundation of China under Grants 61372088, 61271257, and 61328102 and the project funded by the Importation and Development of High-Caliber Talents Project of Beijing Municipal Institutions (CIT\&TCD20130320).

\section{References}

[1] R. Zhang and C. K. Ho, "MIMO broadcasting for simultaneous wireless information and power transfer," IEEE Transactions on Wireless Communications, vol. 12, no. 5, pp. 1989-2001, 2013.

[2] Y. Chen, S. Zhang, S. Xu, and G. Y. Li, "Fundamental trade-offs on green wireless networks," IEEE Communications Magazine, vol. 49, no. 6, pp. 30-37, 2011.

[3] S. Yeh, "Green 4G communications: renewable-energy-based architectures and protocols," in Proceedings of the Global Mobile Congress (GMC '10), pp. 1-5, IEEE, Shanghai, China, October 2010.

[4] J. A. Paradiso and T. Starner, "Energy scavenging for mobile and wireless electronics," IEEE Pervasive Computing, vol. 4, no. 1, pp. 18-27, 2005.

[5] A. M. Zungeru, L. M. Ang, S. Prabaharan, and K. P. Seng, "Radio frequency energy harvesting and management for wireless sensor networks," in Green Mobile Devices and Networks: Energy Optimization and Scavenging Techniques, pp. 341-368, CRC Press, 2012.

[6] R. J. M. Vullers, R. van Schaijk, I. Doms, C. Van Hoof, and R. Mertens, "Micropower energy harvesting," Solid-State Electronics, vol. 53, no. 7, pp. 684-693, 2009.

[7] T. B. Lim, N. M. Lee, and B. K. Poh, "Feasibility study on ambient RF energy harvesting for wireless sensor network," in Proceedings of the IEEE MTT-S International Microwave Workshop Series on RF and Wireless Tecbhnologies for Biomedical and Healthcare Applications (IMWS-BIO '13), Singapore, December 2013.

[8] S. H. Lee, R. Zhang, and K. B. Huang, "Opportunistic wireless energy harvesting in cognitive radio networks," IEEE Transactions on Wireless Communications, vol. 12, no. 9, pp. 4788-4799, 2013.

[9] V. Liu, A. Parks, V. Talla, S. Gollakota, D. Wetherall, and J. R. Smith, "Ambient backscatter: wireless communication out of thin air," in Proceedings of the ACM Conference on SIGCOMM (SIGCOMM '13), pp. 39-50, Hong Kong, China, August 2013.

[10] O. Ozel and S. Ulukus, "Information-theoretic analysis of an energy harvesting communication system," in Proceedings of the 21st IEEE International Symposium on Personal, Indoor and Mobile Radio Communications Workshops (PIMRC Workshops '10), pp. 330-335, IEEE, Instanbul, Turkey, September 2010.

[11] J. Yang and S. Ulukus, "Optimal packet scheduling in an energy harvesting communication system," IEEE Transactions on Communications, vol. 60, no. 1, pp. 220-230, 2012.

[12] Z. Wang, V. Aggarwal, and X. Wang, "Renewable energy scheduling for fading channels with maximum power constraint," in 
Proceedings of the 51st Annual Allerton Conference on Communication, Control, and Computing (Allerton '13), pp. 1394-1400, Monticello, Ill, USA, October 2013.

[13] O. Ozel, K. Tutuncuoglu, J. Yang, S. Ulukus, and A. Yener, "Transmission with energy harvesting nodes in fading wireless channels: optimal policies," IEEE Journal on Selected Areas in Communications, vol. 29, no. 8, pp. 1732-1743, 2011.

[14] Z. Wang and V. Aggarwal, "Power allocation for energy harvesting transmitter with causal information," IEEE Transactions on Communications, vol. 62, no. 11, 2014.

[15] G. E. Forsythe, M. A. Malcolm, and C. B. Moler, Computer Methods for Mathematical Computations, Prentice-Hall, 1976. 


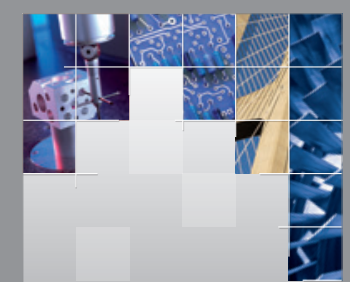

\section{Enfincering}
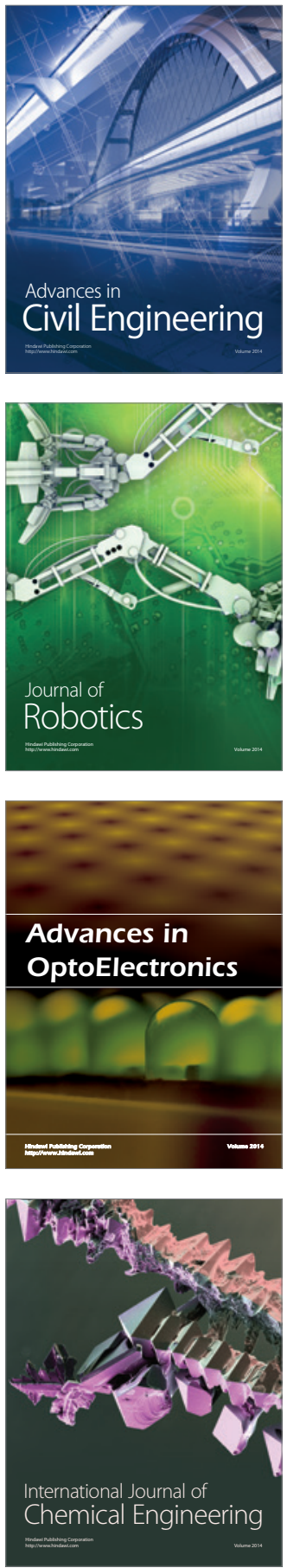

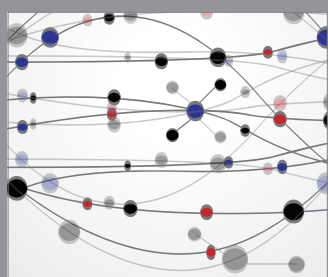

The Scientific World Journal

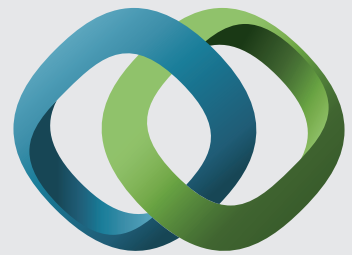

\section{Hindawi}

Submit your manuscripts at

http://www.hindawi.com
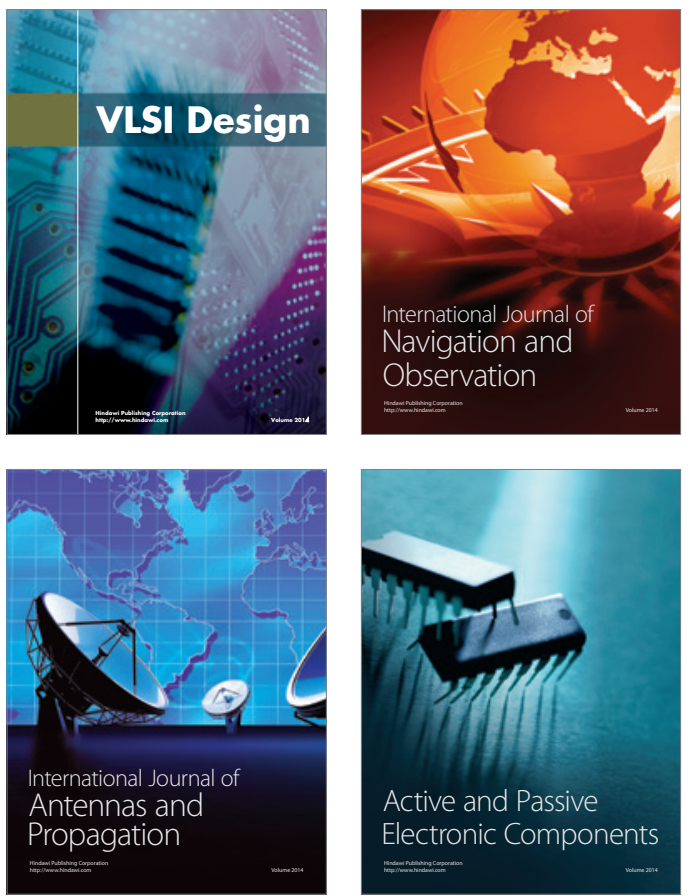
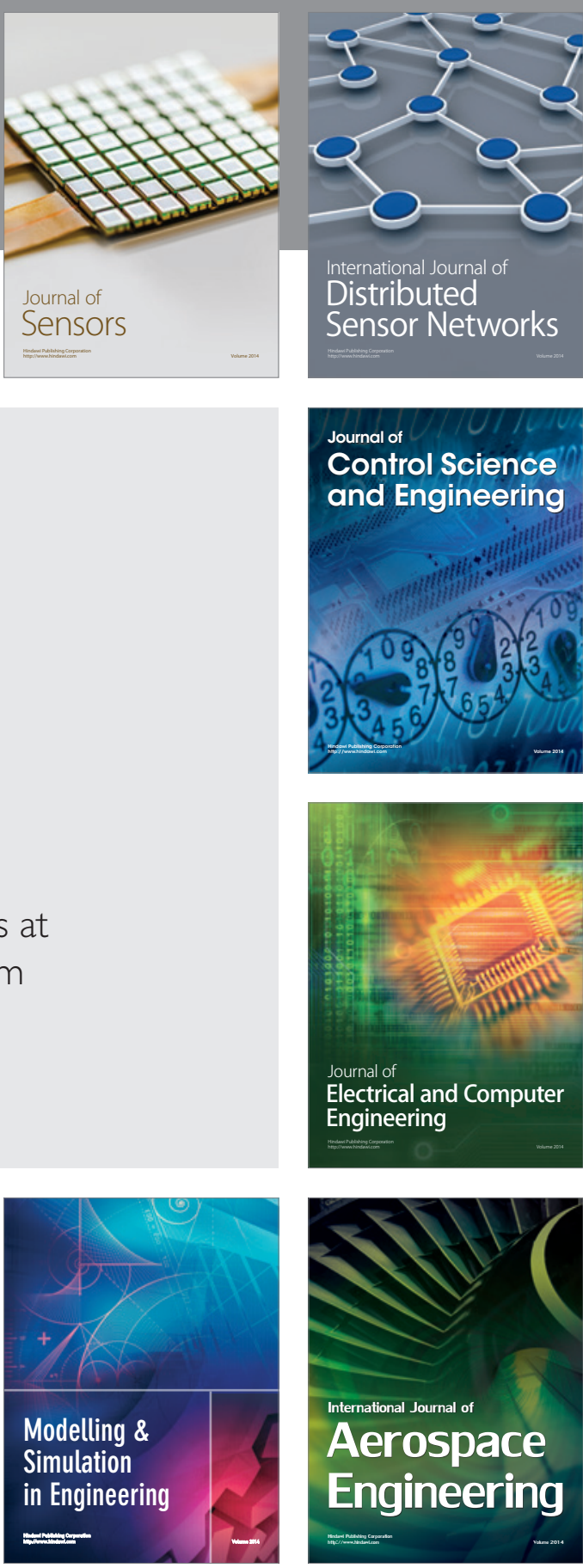

International Journal of

Distributed

Sensor Networks

Journal of

Control Science

and Engineering
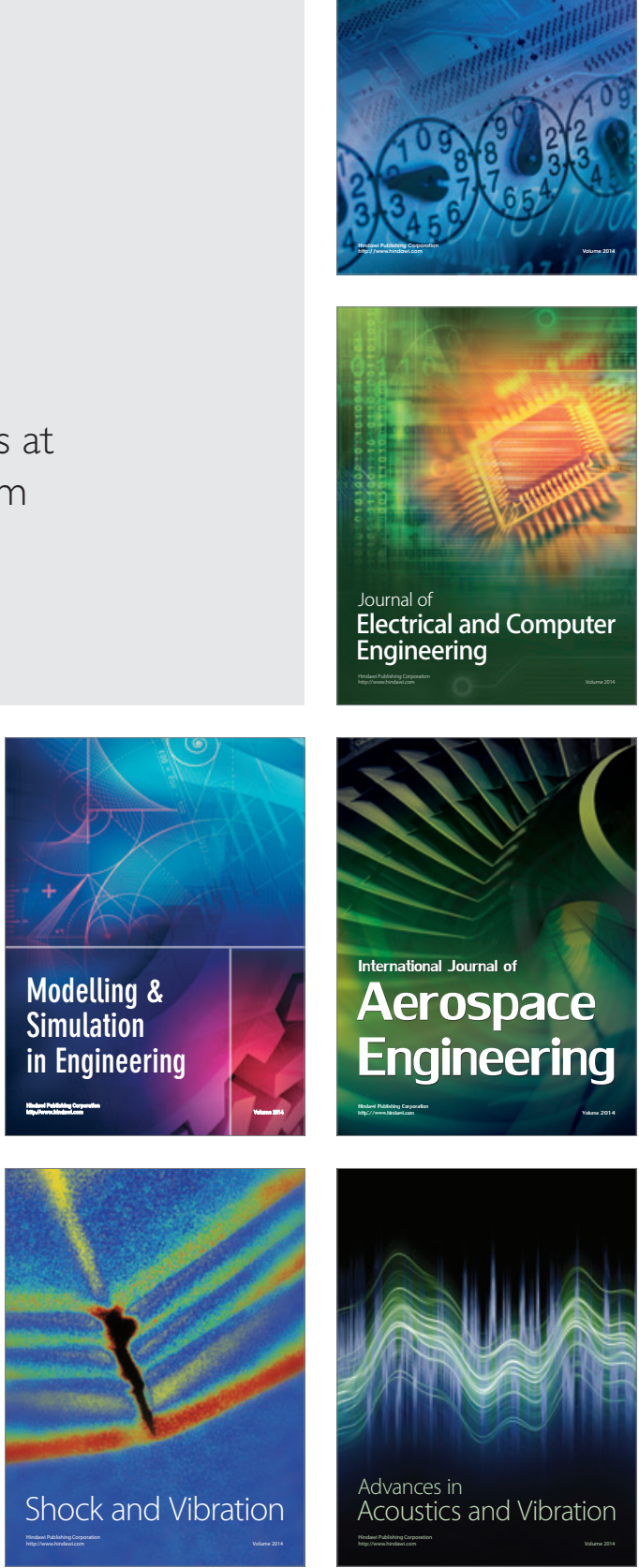